\title{
Synovial Macrophages in Osteoarthritis: The Key to Understanding Pathogenesis?
}

\author{
Amanda Thomson and Catharien M. U. Hilkens* \\ Immunotherapy Research Group, Translational and Clinical Research Institute, Faculty of Medical Sciences, Newcastle \\ University, Newcastle Upon Tyne, United Kingdom
}

OPEN ACCESS

Edited by:

Katharina Schmidt-Bleek,

Charité - Universitätsmedizin Berlin,

Germany

Reviewed by:

Carla R. Scanzello,

University of Pennsylvania,

United States

Simon Mastbergen,

University Medical Center Utrecht,

Netherlands

*Correspondence:

Catharien M. U. Hilkens

catharien.hilkens@newcastle.ac.uk

Specialty section:

This article was submitted to Inflammation,

a section of the journal

Frontiers in Immunology

Received: 10 March 2021

Accepted: 30 April 2021

Published: 15 June 2021

Citation:

Thomson A and Hilkens CMU (2021)

Synovial Macrophages in

Osteoarthritis: The Key to

Understanding Pathogenesis?

Front. Immunol. 12:678757.

doi: 10.3389/fimmu.2021.678757
Effective treatment of osteoarthritis $(\mathrm{OA})$ remains a huge clinical challenge despite major research efforts. Different tissues and cell-types within the joint contribute to disease pathogenesis, and there is great heterogeneity between patients in terms of clinical features, genetic characteristics and responses to treatment. Inflammation and the most abundant immune cell type within the joint, macrophages, have now been recognised as possible players in disease development and progression. Here we discuss recent findings on the involvement of synovial inflammation and particularly the role of synovial macrophages in OA pathogenesis. Understanding macrophage involvement may hold the key for improved OA treatments.

Keywords: osteoarthritis, pathogenesis, macrophage subsets, synovial tissue, inflammation

\section{INTRODUCTION}

Osteoarthritis (OA) is the most common form of arthritis, characterised by pain, swelling and stiffness of the joint. It is also multifactorial in nature, with associated risk factors such as age, sex, ethnicity and obesity. Primary locations affected are synovial joints, including the knee, hip and hands, with knee OA being most frequently observed. OA affects $7 \%$ of the global population and it is estimated that one third of people over the age of 65 suffer with the disease. This equates to approximately 500 million individuals, a figure which has risen by 48\% from 1990-2019 (1-3). Even though OA is a leading cause of disability, the $15^{\text {th }}$ highest cause of years lived with disability globally, no cure or disease modifying treatments are available (1). Symptoms are typically managed through a combination of non-pharmacological methods and non-steroidal anti-inflammatory drugs (NSAIDS). Surgical intervention through joint replacement still remains the only option for end-stage disease, emphasising the need for better treatment strategies. Here, we provide a short overview of the role of inflammation in OA pathogenesis, with a specific focus on the involvement of synovial macrophages. Unravelling the role of these cells may lead to improved stratification of OA patients for anti-inflammatory treatments and/or the identification of novel therapeutic targets. 


\section{OA PATHOGENESIS: CARTILAGE AND SUBCHONDRAL BONE}

OA can affect the entire joint, including cartilage, synovial tissue, subchondral bone and the joint capsule, as well as ligaments and periarticular muscles (4). Cartilage degeneration is probably the most well-known hallmark of $\mathrm{OA}$, and many studies have focused on understanding and preventing its destruction (5). The cartilage provides an important lubricated covering to the bone surfaces where the femur, tibia and patella articulate with each other. This absorbs stress created during movement and importantly, creates a smooth platform to allow for efficient joint motions. It comprises an extracellular matrix (ECM), composed mostly of type II collagen and aggrecan proteoglycans, which bring strength and flexibility to the tissue respectively $(6,7)$.

Chondrocytes constitute the cellular component (7) and maintain homeostasis through synthesis and degradation of the cartilage proteins. In OA, this equilibrium shifts to catabolism and chondrocytes adopt an activated state characterised by increased cell proliferation, molecular alterations and production of ECM degrading enzymes. This leads to cartilage damage (8-10). MMP family proteins and aggrecanases, the most widely studied ECM degrading enzymes, are both able to degrade native collagen and aggrecan (11-13). This breakdown of the ECM leads to fibrillation and subsequent fissure development within the cartilage layers and as a result, the subchondral bone (SB), situated directly beneath, is exposed to the articular cavity. The joint therefore becomes unable to function normally with regards to gliding movements and is incapable of effectively absorbing mechanical stress. Composed of the SB plate and underlying trabecular and subarticular bone, the SB functions as a shock absorber and helps distribute the mechanical load of the joint. Structural changes to SB can also be seen in OA, and include increased bone turnover, the development of microfractures and increased angiogenesis. Bone sclerosis, osteophytes, bone cysts and bone marrow lesions, detected via MRI can also be seen.

Inflammatory mediators have long been known to play a role in the breakdown of the cartilage ECM. In particular, OA patients have increased levels of IL- $1 \beta$, TNF- $\alpha$ and IL-6. IL- $1 \beta$ is an essential mediator of joint inflammation and its overexpression by chondrocytes can be seen in early osteoarthritic cartilage (14, 15). Such levels cause an abnormal chondrocyte phenotype, which directly interferes with the synthesis of ECM collagen and aggrecan proteins. An associated increased release of MMP and aggracanase enzymes such as MMP-1, MMP-3 and MMP-13 is also seen, with destructive effects on cartilage components $(16,17)$. Functioning in an autocrine manner, IL-1 $\beta$ can induce its own secretion and stimulate the synthesis of other inflammatory mediators, again such as TNF- $\alpha$ and IL-6. Often found working in synergy with IL$1 \beta$, TNF- $\alpha$ binding to its receptors induces a similar NF- $\kappa B$ signalling cascade to increase inflammation and catabolism through enhancing adhesion molecule expression, the synthesis of further cytokines, and promoting the expression of more MMP family enzymes able to degrade the $\operatorname{ECM}(18,19)$. Other cytokines such as IL-8, IL-18, IL-17 and IL-22 are increased when comparing human inflamed and non-inflamed synovial tissue (20). In particular such cytokines are associated with Th17 and NK22 cells, along with the recruitment of neutrophils into the tissue, all of which are capable of further promoting synovitis (20). IL-6, produced by cells such as chondrocytes, osteoblasts, fibroblasts, macrophages and adipocytes can also synergise with other cytokines to affect the ECM. However, evidence indicates IL-6 is the key cytokine to affect the SB layer of the joint by promoting the formation of osteoclasts to increase bone absorption within the joint $(21,22)$. Also associated with the bone itself, high TGF- $\beta$ has been reported via in vitro and in vivo studies to promote the production of osteophytes, as well as increasing chondrocyte hypertrophy via alternative signalling pathways (23). Further descriptions of cytokines involved in OA are beyond the scope of this mini review, but such information has been discussed elsewhere (24).

\section{OA PATHOGENESIS: SYNOVIAL TISSUE}

The synovium lines the joint cavity. Its main function is to produce synovial fluid to equip the joint for efficient movement. Concentrations of synovial fluid components (lubrican and hyaluronic acid) are often altered in OA, influencing cartilage integrity. The synovium is composed of two main regions: the lining and sublining layers. Synovial lining consists mainly of macrophage and fibroblast cell types. The sublining contains additional fibroblasts, macrophages, adipose cells and blood vessels, with low numbers of lymphocytes also detectable (25).

Low-grade synovial inflammation has been observed in over half of OA patients at both early and late stages of disease (26-33) which has led to the notion that OA is not simply caused by an age-related wear and tear of the joint. Unlike more typical inflammatory arthritides [e.g., rheumatoid arthritis (RA)] OA synovitis is usually not accompanied by overt systemic inflammation. In RA, the inflamed synovium is characterised by vasculitis and a mixed immune cell infiltrate. This infiltrate predominantly consists of lymphocytes but also includes myeloid cells such as macrophages. Inflammation and angiogenesis in RA are further exacerbated due to antigen presentation and cytokine release. Subsequently, cartilage degradation and bone erosion arises over time in response to protease (e.g. MMPs) and cytokine release (e.g. TNF- $\alpha$ and IL-6) (34). A role for macrophages, with their associated inflammatory cytokines (IL-6 and TNF- $\alpha$ ), has also been recognised in RA (35).

In $\mathrm{OA}$, synovial inflammation is less pronounced, but there is ample evidence to support its pathogenic role $(26,30,36)$. Histopathological studies since the 1980s have identified inflammatory signatures (cellular hyperproliferation, increased angiogenesis and lymphocyte aggregate appearance) within OA synovium (37-39). The degree of inflammation is highly heterogenous between patients, but nevertheless, has been associated with pain and disease progression. As highlighted in the previous section, inflammatory molecules, including IL- $1 \beta$ and TNF- $\alpha$, are able to induce protease secretion by chondrocytes, highlighting possible crosstalk between the synovium and other 
joint tissues (40). It has also been reported that the quantity of activated macrophages within patient OA synovium correlates with disease severity and progression (41). Synovitis confers a 9fold greater risk of individuals presenting with painful knee OA (30). Elevated inflammatory markers have been detected in the serum and synovial fluid of OA patients and levels of serum TNF$\alpha$ correlate with OA kellgren-lawrence X-ray grades (42). However, whilst treatments to dampen inflammation in RA have shown success (43), trials with anti-inflammatory drugs in OA have been disappointing thus far. A possible reason may be that the inflammatory players and processes differ greatly between patients; a notion that is supported by heterogeneity of the immune cell infiltrate in the OA synovium. For example, bicompartmental OA is characterised by higher infiltration of $\mathrm{CD} 4^{+} \mathrm{T}$ cells into the synovium than uni-compartmental disease (44) and we recently demonstrated highly variable numbers of macrophages as well as other immune cell subsets in the OA synovium (45). Therefore, we argue that a better understanding of the inflammatory players in OA would benefit the development of improved therapeutic strategies; either through stratification of OA patients for the most suitable disease-modifying treatments and/or the identification of novel targets.

\section{SYNOVIAL MACROPHAGES}

The most abundant immune cell type in the OA synovium is the macrophage. Macrophages are often described as displaying an M1 or M2 phenotype. Activated by environmental factors such as IFN- $\gamma$, TNF- $\alpha$ and LPS, M1 macrophages secrete proinflammatory cytokines and low levels of IL-10. M2 macrophages display an anti-inflammatory profile and possess tissue-repair functions (46). However, such M1/M2 descriptions are now regarded as extreme poles of a spectrum in many fields, with macrophage phenotype varying greatly depending on the tissue environment. High macrophage numbers are detected in OA patients compared to healthy controls and quantities of activated macrophages correlate with clinical symptoms $(41,47)$. Furthermore, increases in macrophage associated molecules (sCD163 and sCD14) and chemoattractants such as CCL2 and $\mathrm{CX}_{3} \mathrm{CL}_{1}$ in $\mathrm{OA}$ patient synovial fluid are linked with clinical outcome in OA $(48,49)$. It is thought that synovial macrophages respond to danger-associated molecular associated patterns, including cartilage fragments and intracellular proteins from necrotic cells, consequently contributing to cartilage damage and bone alterations through the release of cytokines such as IL-1 TNF- $\alpha$ and TGF- $\beta$ (Figure 1). In support of this, in vitro studies have highlighted that depletion of $\mathrm{CD} 14^{+}$macrophages from synovial cell cultures results in a reduction of IL- $1 \beta$, TNF- $\alpha$, MMPs and aggrecanase enzymes which are able to degrade joint cartilage (50). Latest research investigating joint macrophages under normal and disease conditions (RA and OA) has led to the identification of multiple synovial macrophage subsets in the same joint. In a setting were inflammation aids disease, it's probable that the abundance of distinct macrophage subsets could perpetuate or indeed help to resolve OA.

\section{NOVEL SYNOVIAL MACROPHAGE SUBSETS IN INFLAMMATORY ARTHRITIS}

To carry out comprehensive investigations of joint macrophages Culemann and colleagues utilised transgenic mice and models of inflammatory arthritis (51). Exploring the origin of increased

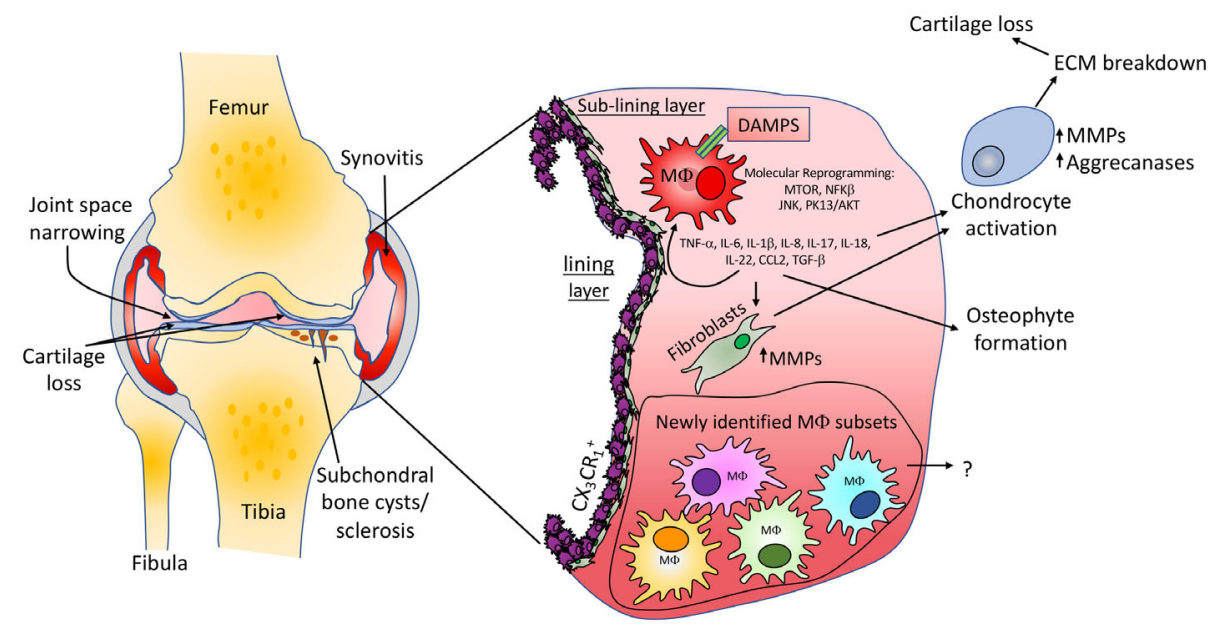

FIGURE 1 | Knee osteoarthritis pathology and macrophage involvement. Common features of OA including cartilage loss, narrowing of the joint space, synovitis and the development of subchondral bone cysts and sclerosis are shown. Macrophages in the synovium can contribute to OA via the release of inflammatory molecules which are able to stimulate resident fibroblast populations to produce cartilage extracellular matrix degrading enzymes. Inflammatory molecules are also able to activate chondrocytes, promoting an abnormal molecular and cellular phenotype, again promoting cartilage loss. We suggest that the same or similar macrophage populations newly identified in inflammatory arthritis studies will be found in OA tissues, may differ between clinical states and could provide therapeutic targets for subgroups of patients. The identification and impact of such populations in OA development is yet to be determined. 
macrophage numbers during arthritis, the authors identified a $\mathrm{CX}_{3} \mathrm{CR}_{1}+$ macrophage subset in direct proximity with collagen VI expressing synovial fibroblast cells. This subset expressed tight junctional protein markers usually associated with that of endothelial cells (F11r, ZO-1 and claudin-5), was maintained by a distinct $\mathrm{Ki} 67+\mathrm{CX}_{3} \mathrm{CR}_{1}$ - interstitial macrophage population and formed a dense barrier between the synovial capillary network and the intra-articular space. At the onset of arthritis $\mathrm{CX}_{3} \mathrm{CR}_{1}+$ macrophages underwent altered morphology, and their cell-to-cell contacts were abrogated. Consequently, there was a breakdown of the "macrophage barrier" and $\mathrm{CX}_{3} \mathrm{CR}_{1}$ macrophage populations were found to rapidly proliferate in response. Coincidingly blood-derived macrophages infiltrated the tissue. The $\mathrm{CX}_{3} \mathrm{CR}_{1}+$ macrophage subset may therefore promote an important synovial regulatory function to seclude and protect intra-articular structures. Work to identify macrophage populations capable of promoting a "macrophage barrier" and the downstream implications of this in human OA could prove to be highly advantageous. Comparing RNA sequencing of mouse macrophage populations the authors also showed that $\mathrm{CX}_{3} \mathrm{CR}_{1}+$ macrophages expressed immune related genes (TREM2 and VSIG4) and that additional heterogeneity existed within $\mathrm{CX}_{3} \mathrm{CR}_{1}$ - macrophages (51). This is a significant observation as mouse macrophage expression profiles correlate with recent sc-RNA sequencing data sets from human RA patients. Exploring human synovial macrophages within RA, and using OA tissue as a comparison, Zhang and colleagues identified four transcriptionally distinct subsets (SCM1-M4) (52). In particular, the presence of IL- $1 \beta+$ pro-inflammatory macrophages (SCM1 subset) were upregulated in "leukocyterich" RA tissue compared to OA samples. Conversely, a SCM2 subset, which express VSIG4 similar to mouse $\mathrm{CX}_{3} \mathrm{CR}_{1}+$ macrophages, were upregulated in $\mathrm{OA}$, suggesting they too are a resident synovial population.

In line with this, Alivernini and colleagues recently identified that clinically distinct states of RA can be characterised by relative proportions of particular macrophage populations. Firstly, healthy donor macrophages and those from patients in remission phenotypically were MerTK+ and CD206+. Patients with active RA had higher amounts of MerTK- CD206- and fewer MerTK+ CD206+ macrophages (53). Delving further to unravel the heterogeneity of these two populations, sc-RNA sequencing revealed nine distinct synovial macrophage clusters that could be classified into again four subpopulations: TREM2+, FOLR2 ${ }^{\text {high }}, \mathrm{HLA}+$ and $\mathrm{CD} 48+$. Comparing relative gene ontology pathways of the nine macrophage clusters with clinical state revealed that MerTK+ TREM2+ and MerTK+ FOLR2+ macrophages were predominantly in healthy tissue. They also showed gene expression (ALDH1A1 and VSIG4) that would promote regulation of adaptive immunity through inhibition of $\mathrm{T}$ effector cells. Patients with sustained remission showed an increase in MerTK+ FOLR2 $2^{\text {high }}$ LYVE1+ macrophages which link this subset to tissue remodelling and homeostasis. In comparison, treatment naïve and active RA had increased proportions of MerTK- CD48- SPP1+ and MerTKCD48- S100A12+ clusters which showed a pro-inflammatory transcriptome phenotype. The authors further showed that the MerTK- CD206- and MerTK+ CD206+ macrophage clusters were able to induce proinflammatory responses and repair synovial fibroblasts phenotypes, respectively. An overview of all novel synovial macrophages can be seen in Table $\mathbf{1}$.

\section{SYNOVIAL MACROPHAGE SUBSETS IN OA}

Macrophages in OA are not completely understood and most studies to date refer to macrophages as M1/M2, as comprehensively reviewed by Fernandes and colleagues (54). M1 macrophages in OA are linked with destructive processes: down regulation of collagen type II and aggrecan synthesis, and upregulation of enzymes such as MMP-1, -3, -9 and -13 (55). In comparison, there is murine evidence that "tissue repairing" M2 macrophage associated cytokines IL-4 and IL-10 are induced with moderate physical activity within the OA synovium, potentially promoting a protective environment (56). Macrophage-related chemokine CCL2, produced in response to inflammatory stimuli by chondrocytes, and its receptor CCR2 has also been noted. Depletion of CCL2/CCR2 is associated with decreased pain severity and older knockout mice show reduced structural disease after joint de-stabilisation (57). The upstream inducer of CCL2, TGF- $\alpha$ has been identified as a possible gene candidate for determining human OA risk and cartilage thickness. TGF- $\alpha$ inhibition in models also shows reduced structural disease, making this an interesting macrophagerelated therapeutic target (58). In other mouse studies, contradictory results are reported. Macrophage depletion has been shown to reduce OA symptoms such as osteophyte formation in some cases, but in others increased synovitis can be seen due to $\mathrm{CD}^{+} \mathrm{T}$ cell and neutrophil infiltration $(59,60)$. Discrepancies of macrophage manipulation in mouse models however could relate to the mechanism of disease onset used e.g., obesity, surgical etc., something which may suggest the presence of possible OA phenotypes.

With new knowledge of RA synovial macrophage subsets emerging that goes beyond the classical M1/M2 concept, there is further potential to generate new therapeutic strategies to promote the resolution of synovitis in OA, specifically targeting patients that would likely benefit most. Whether the same cellular populations and mechanisms exist in OA remains unclear and is currently under investigation. Nevertheless, the first study to explore and characterise the cellular and transcriptional heterogeneity on a single cell level in matched synovial and cartilage from OA patients was recently published by the Kraus laboratory (61). Here the authors identify twelve synovial cellular populations, including two distinct macrophage populations, and show that key OA mediators (TNF, IL-6 and IL-1 $\beta$ ) are released into the joint space via HLA-DRA+ macrophages and DCs. Cytokine expression was 25-fold higher within the synovium compared to damaged cartilage areas and no cytokine was exclusively expressed by chondrocytes themselves. This emphasises the possible crosstalk between 
TABLE 1 | Recently Identified novel macrophage subsets.

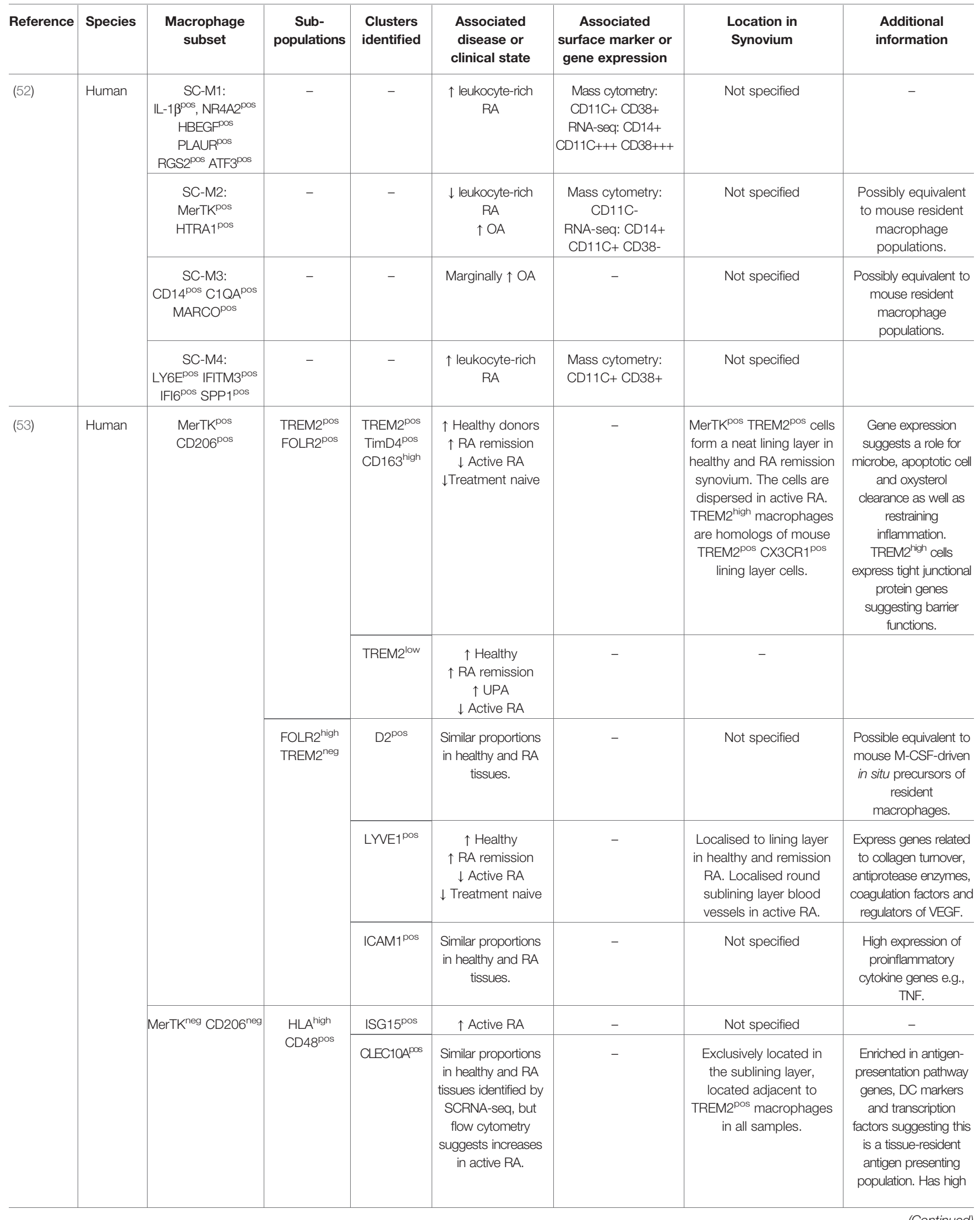

(Continued) 
TABLE 1 | Continued

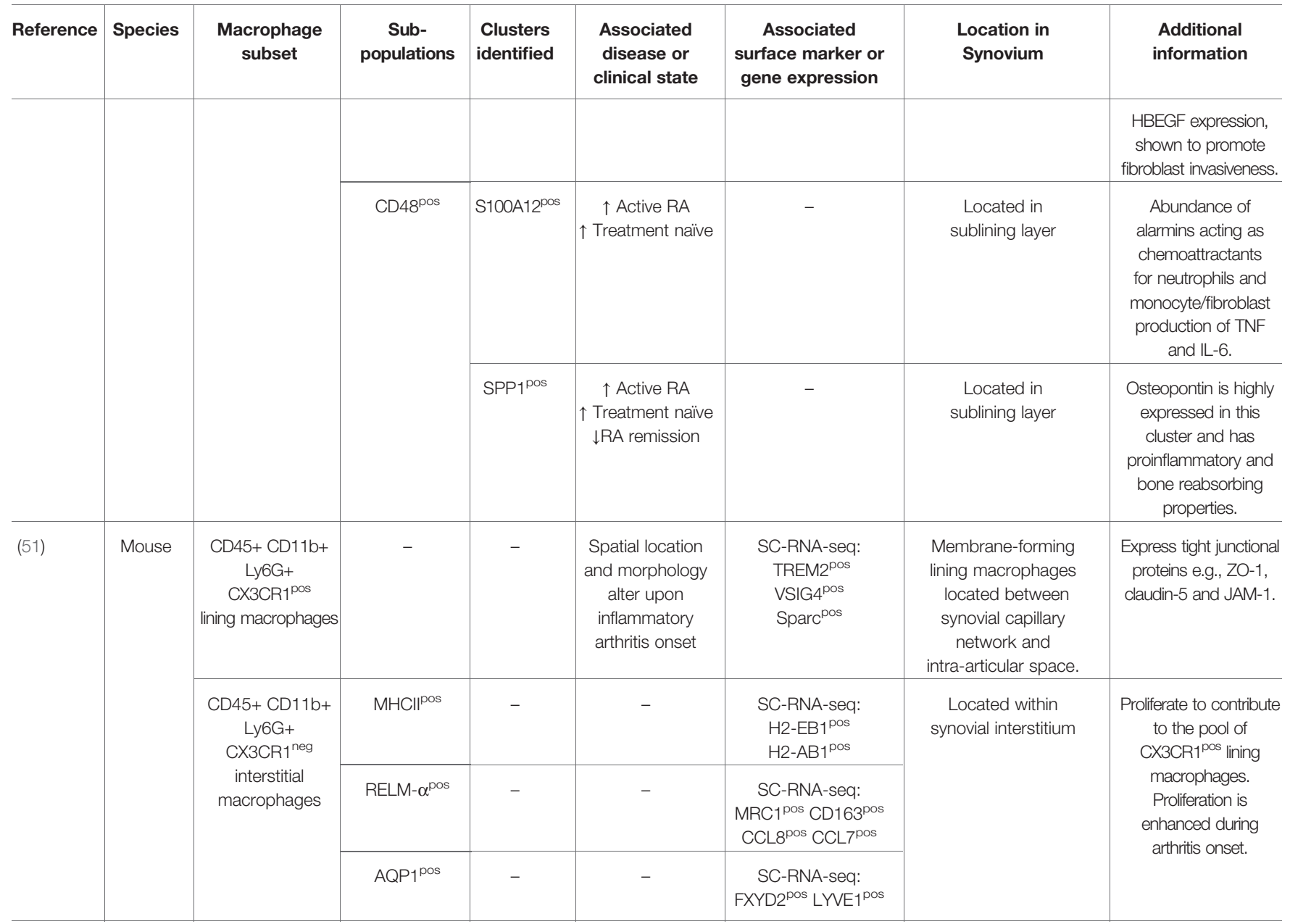

joint tissues in OA development. Such cytokine upregulation in the synovium may have synergistic effects on signalling pathways within other joint tissues to increase inflammation and in turn promote cartilage breakdown (62). This study again displays a potential for tissue specific targeting of pathogenic molecules or cells within the synovium itself in order to treat OA (61).

A recent study from our own laboratory identified distinct human knee OA endotypes (where insights of the pathogenic mechanism of disease are given) based on gene expression profiles of synovial macrophages. One of these endotypes displayed increased numbers of synovial CD14+ macrophages that closely aligned with synovial macrophages from inflammatory arthritis patients and displayed a cell proliferation signature and high Ki67 expression (45). However, whether this finding is in any way comparable with Ki67-expressing $\mathrm{CX}_{3} \mathrm{CR}_{1}$ - macrophages in mice remains to be answered. The discovery of multiple synovial macrophage subsets may help to explain the contradicting results derived from in vivo macrophage-depletion studies of $\mathrm{OA}$, and it is thought that macrophage subset identification could be used to aid the stratification of patients for treatment. Understanding the impact of specific synovial macrophage subsets is a research priority, with fundamental questions remaining. Do the same macrophage subsets exist in OA as in RA? Do macrophage subsets differ between OA disease stages? Do particular macrophage subsets associate with clinical symptoms? And importantly, what other cells within the synovial environment do macrophage subsets communicate with or influence? Unearthing such information could prove crucial for understanding $\mathrm{OA}$ pathogenesis and importantly reveal new therapeutic targets. Identification of several macrophage subsets within joint tissue truly advocates for an alternative assessment of how this cellular population is involved in OA.

\section{FUTURE DIRECTIONS}

New directions for OA research are imperative as clinical trials for disease-modifying treatments thus far have been largely disappointing. Disease heterogeneity often is suggested as a possible explanation. In 2016 Dell'Isola and colleagues provided evidence for the existence of six major OA clinical phenotypes (where observable traits are used to define disease clusters), reporting that $84 \%$ of subjects across twenty-four studies could be classified in this manner (63). 12\% of OA patients could be classified into an "inflammatory" phenotype, 
whilst the others were characterised into chronic pain, metabolic syndrome, bone and cartilage metabolism, mechanical overload and minimal disease phenotypes. Regardless of such classifications, trials of anti-IL-1 agents that specifically focused on patients with synovitis (the inflammatory phenotype) still resulted in limited improvement in pain scores and synovial inflammation (64). This implies factors other than IL-1 are at play in this patient subgroup. The ability to further classify patients more effectively could significantly transform and enhance OA clinical trial efficiency. Such approaches have already been applied in other settings such as in RA and asthma, as a method for identifying "clinicopathobiologic clusters" (65-67). By selecting patients based on particular OA molecular features, such as signalling pathways or other distinct molecular mechanisms as opposed to only by clinical phenotype, such as the presence of synovitis, patient subgroups most likely to benefit from particular therapies may be more easily identified. Of course, revealing molecular endotypes of OA is an extremely complex task. Advancements in imaging

\section{REFERENCES}

1. Hunter DJ, March L, Chew M. Osteoarthritis in 2020 and Beyond: A Lancet Commission. Lancet (2020) 396:1711-2. doi: 10.1016/10.1001/ jamapediatrics.2020.4573

2. Hunter DJ, Bierma-Zeinstra S. Osteoarthritis. Lancet (2019) 393:1745-59. doi: 10.1016/S0140-6736(19)30417-9

3. Martel-Pelletier J, Barr AJ, Cicuttini FM, Conaghan PG, Cooper C, Goldring MB, et al. Osteoarthritis. Nat Rev Dis Primers (2016) 2:1-18. doi: 10.1038/ nrdp.2016.72

4. Loeser RF, Goldring SR, Scanzello CR, Goldring MB. Osteoarthritis: A Disease of the Joint as An Organ. Arthritis Rheum (2012) 64(6):1697-707. doi: 10.1002/art.34453

5. Young DA, Barter MJ, Wilkinson DJ. Recent Advances in Understanding the Regulation of Metalloproteinases. F1000 Res (2019) 8:195. doi: 10.12688/ f1000research.17471.1

6. Buckwalter JA, Mankin HJ. Articular Cartilage: Tissue Design and Chondrocyte-Matrix Interactions. Instr Course Lectures (1998) 47:477-86. doi: 10.2106/00004623-199704000-00021

7. Bhosale AM, Richardson JB. Articular Cartilage: Structure, Injuries and Review of Management. Br Med Bull (2008) 87:77-95. doi: 10.1093/bmb/ ldn025

8. Goldring MB, Marcu KB. Cartilage Homeostasis in Health and Rheumatic Diseases. Arthritis Res Ther (2009) 11:224. doi: 10.1186/ar2592

9. Katsara O, Attur M, Ruoff R, Abramson SB, Kolupaeva V. Increased Activity of the Chondrocyte Translational Apparatus Accompanies Osteoarthritic Changes in Human and Rodent Knee Cartilage. Arthritis Rheumatol (2017) 69(3):586-97. doi: 10.1002/art.39947

10. Mitchell PG, Magna HA, Reeves LM, Lopresti-Morrow LL, Yocum SA, Rosner PJ, et al. Cloning, Expression, and Type II Collagenolytic Activity of Matrix Metalloproteinase-13 From Human Osteoarthritic Cartilage. J Clin Invest (1996) 97(3):761-8. doi: 10.1172/JCI118475

11. Sandy JD. A Contentious Issue Finds Some Clarity: On the Independent and Complementary Roles of Aggrecanase Activity and MMP Activity in Human Joint Aggrecanolysis. Osteoarthr Cartil (2006) 14:95-100. doi: 10.1016/ j.joca.2005.09.004

12. Rengel Y, Ospelt C, Gay S. Proteinases in the Joint: Clinical Relevance of Proteinases in Joint Destruction. Arthritis Res Ther (2007) 9:22. doi: 10.1186/ ar2304

13. Cawston TE, Wilson AJ. Understanding the Role of Tissue Degrading Enzymes and Their Inhibitors in Development and Disease. Best Pract Res Clin Rheumatol (2006) 20:983-1002. doi: 10.1016/j.berh.2006.06.007 techniques, identification of novel OA biomarkers and increased knowledge of cellular communications (like that of macrophage subsets) within joint tissues will be of great importance. Ultimately, this approach could facilitate the development of better treatment strategies for OA patients.

\section{AUTHOR CONTRIBUTIONS}

$\mathrm{AT}$ and $\mathrm{CH}$ conceived the idea of the manuscript and performed literature searches. AT wrote the manuscript. $\mathrm{CH}$ reviewed and edited the manuscript. All authors contributed to the article and approved the submitted version.

\section{FUNDING}

This work was funded by the JGW Patterson Foundation.

14. Fan Z, Bau B, Yang H, Soeder S, Aigner T. Freshly Isolated Osteoarthritic Chondrocytes are Catabolically More Active Than Normal Chondrocytes, But Less Responsive to Catabolic Stimulation With Interleukin-1? Arthritis Rheum (2005) 52(1):136-43. doi: 10.1002/art.20725

15. Towle CA, Hung HH, LJ B, Treadwell BV, Mangham DC. Detection of Interleukin-1 in the Cartilage of Patients With Osteoarthritis: A Possible Autocrine/Paracrine Role in Pathogenesis. Osteoarthr Cartil (1997) 5(5):293300. doi: 10.1016/S1063-4584(97)80008-8

16. Vincenti MP, Brinckerhoff CE. Transcriptional Regulation of Collagenase (MMP-1, MMP-13) Genes in Arthritis: Integration of Complex Signalling Pathways for the Recruitment of Gene-Specific Transcription Factors. Arthritis Res (2002) 4:157-64. doi: 10.1186/ar401

17. Mengshol JA, Vincenti MP, Coon CI, Barchowsky A, Brinckerhoff CE. Interleukin-1 Induction of Collagenase 3 (Matrix Metalloproteinase 13) Gene Expression in Chondrocytes Requires p38, C-Jun N-terminal Kinase, and Nuclear Factor $\kappa \mathrm{B}$ : Differential Regulation of Collagenase 1 and Collagenase 3. Arthritis Rheum (2000) 43(4):801-11. doi: 10.1002/15290131(200004)43:4<801::AID-ANR10>3.0.CO;2-4

18. Roman-Blas JA, Jimenez SA. Nf- $\kappa$ B as A Potential Therapeutic Target in Osteoarthritis and Rheumatoid Arthritis. Osteoarthr Cartil (2006) 14:839-48. doi: 10.1016/j.joca.2006.04.008

19. Marcu K B, Otero M, Olivotto E, Maria Borzi R, B. Goldring M. NF- $\kappa B$ Signaling: Multiple Angles to Target OA. Curr Drug Targets (2010) 11(5):599613. doi: $10.2174 / 138945010791011938$

20. Deligne C, Casulli S, Pigenet A, Bougault C, Campillo-Gimenez L, Nourissat $\mathrm{G}$, et al. Differential Expression of Interleukin-17 and Interleukin-22 in Inflamed and Non-Inflamed Synovium From Osteoarthritis Patients. Osteoarthr Cartil (2015) 23(11):1843-52. doi: 10.1016/j.joca.2014.12.007

21. Steeve KT, Marc P, Sandrine T, Dominique H, Yannick F. IL-6, RANKL, TNF-Alpha/IL-1: Interrelations in Bone Resorption Pathophysiology. Cytokine Growth Factor Rev (2004) 15:49-60. doi: 10.1016/j.cytogfr. 2003.10.005

22. Chenoufi HL, Diamant M, Rieneck K, Lund B, Stein GS, Lian JB. Increased mRNA Expression and Protein Secretion of Interleukin-6 in Primary Human Osteoblasts Differentiated In Vitro From Rheumatoid and Osteoarthritic Bone. J Cell Biochem (2001) 81(4):666-78. doi: 10.1002/jcb.1104

23. van der Kraan PM. Differential Role of Transforming Growth Factor-Beta in an Osteoarthritic or a Healthy Joint. J Bone Metab (2018) 25(2):65. doi: 10.11005/jbm.2018.25.2.65

24. Chow YY, Chin K-Y. The Role of Inflammation in the Pathogenesis of Osteoarthritis. Mediators Inflamm (2020) 2020:8293921. doi: 10.1155/2020/ 8293921 
25. Smith M D. The Normal Synovium. Open Rheumatol J (2012) 5(1):100-6. doi: $10.2174 / 1874312901105010100$

26. Haywood L, McWilliams DF, Pearson CI, Gill SE, Ganesan A, Wilson D, et al. Inflammation and Angiogenesis in Osteoarthritis. Arthritis Rheum (2003) 48 (8):2173-7. doi: 10.1002/art.11094

27. Benito MJ, Veale DJ, FitzGerald O, Van Den Berg WB, Bresnihan B. Synovial Tissue Inflammation in Early and Late Osteoarthritis. Ann Rheum Dis (2005) 64(9):1263-7. doi: 10.1136/ard.2004.025270

28. Torres L, Dunlop DD, Peterfy C, Guermazi A, Prasad P, Hayes KW, et al. The Relationship Between Specific Tissue Lesions and Pain Severity in Persons With Knee Osteoarthritis. Osteoarthr Cartil (2006) 14(10):1033-40. doi: 10.1016/j.joca.2006.03.015

29. Hill CL, Hunter DJ, Niu J, Clancy M, Guermazi A, Genant H, et al. Synovitis Detected on Magnetic Resonance Imaging and Its Relation to Pain and Cartilage Loss in Knee Osteoarthritis. Ann Rheum Dis (2007) 66(12):1599603. doi: 10.1136/ard.2006.067470

30. Baker K, Grainger A, Niu J, Clancy M, Guermazi A, Crema M, et al. Relation of Synovitis to Knee Pain Using Contrast-Enhanced MRIs. Ann Rheum Dis (2010) 69(10):1779-83. doi: 10.1136/ard.2009.121426

31. Conaghan PG, D'Agostino MA, Le Bars M, Baron G, Schmidely N, Wakefield $\mathrm{R}$, et al. Clinical and Ultrasonographic Predictors of Joint Replacement for Knee Osteoarthritis: Results From a Large, 3-Year, Prospective EULAR Study. Ann Rheum Dis (2010) 69(4):644-7. doi: 10.1136/ard.2008.099564

32. Roemer FW, Guermazi A, Felson DT, Niu J, Nevitt MC, Crema MD, et al. Presence of MRI-Detected Joint Effusion and Synovitis Increases the Risk of Cartilage Loss in Knees Without Osteoarthritis at 30-Month Follow-Up: The MOST Study. Ann Rheum Dis (2011) 70(10):1804-9. doi: 10.1136/ ard.2011.150243

33. Scanzello CR, McKeon B, Swaim BH, Dicarlo E, Asomugha EU, Kanda V, et al. Synovial Inflammation in Patients Undergoing Arthroscopic Meniscectomy: Molecular Characterization and Relationship to Symptoms. Arthritis Rheum (2011) 63(2):391-400. doi: 10.1002/art.30137

34. Smolen JS, Aletaha D, McInnes IB. Rheumatoid Arthritis. Lancet (2016) 388:2023-38. doi: 10.1016/S0140-6736(16)30173-8

35. Kurowska-Stolarska M, Alivernini S. Synovial Tissue Macrophages: Friend or Foe? RMD Open (2017) 3:e000527. doi: 10.1136/rmdopen-2017-000527

36. Mathiessen A, Conaghan PG. Synovitis in Osteoarthritis: Current Understanding With Therapeutic Implications. Arthritis Res Ther (2017) 19:18. doi: 10.1186/s13075-017-1229-9

37. Goldenberg DL, Egan MS, Cohen AS. Inflammatory Synovitis in Degenerative Joint Disease. J Rheumatol (1982) 9(2):204-9.

38. Lindblad S, Hedfors E. Arthroscopic and Immunohistologic Characterization of Knee Joint Synovitis in Osteoarthritis. Arthritis Rheum (1987) 30(10):10818. doi: 10.1002/art.1780301001

39. Revell PA, Mayston V, Lalor P, Mapp P. The Synovial Membrane in Osteoarthritis: A Histological Study Including the Characterisation of the Cellular Infiltrate Present in Inflammatory Osteoarthritis Using Monoclonal Antibodies. Ann Rheum Dis (1988) 47(4):300-7. doi: 10.1136/ard.47.4.300

40. Milner JM, Kevorkian L, Young DA, Jones D, Wait R, Donell ST, et al. Fibroblast Activation Protein Alpha Is Expressed by Chondrocytes Following a Pro-Inflammatory Stimulus and Is Elevated in Osteoarthritis. Arthritis Res Ther (2006) 8(1):R23. doi: 10.1186/ar1877

41. Kraus VB, McDaniel G, Huebner JL, Stabler TV, Pieper CF, Shipes SW, et al. Direct In Vivo Evidence of Activated Macrophages in Human Osteoarthritis. Osteoarthr Cartil (2016) 24(9):1613-21. doi: 10.1016/j.joca.2016.04.010

42. Özler K, Aktas E, Atay C, Yilmaz B, Arikan M, Güngör S. Serum and Knee Synovial Fluid Matrix Metalloproteinase-13 and Tumor Necrosis FactorAlpha Levels in Patients With Late-Stage Osteoarthritis. Acta Orthop Traumatol Turc (2016) 50(3):356-61. doi: 10.1016/j.aott.2015.11.003

43. Burmester GR, Pope JE. Novel Treatment Strategies in Rheumatoid Arthritis. Lancet (2017) 389:2338-48. doi: 10.1016/S0140-6736(17)31491-5

44. Moradi B, Rosshirt N, Tripel E, Kirsch J, Barié A, Zeifang F, et al. Unicompartmental and Bicompartmental Knee Osteoarthritis Show Different Patterns of Mononuclear Cell Infiltration and Cytokine Release in the Affected Joints. Clin Exp Immunol (2015) 180(1):143-54. doi: 10.1111/ cei. 12486

45. Wood MJ, Leckenby A, Reynolds G, Spiering R, Pratt AG, Rankin KS, et al. Macrophage Proliferation Distinguishes 2 Subgroups of Knee
Osteoarthritis Patients. JCI Insight (2019) 4(2):e125325. doi: 10.1172/ jci.insight. 125325

46. Mills CD, Kincaid K, Alt JM, Heilman MJ, Hill AM. M-1/M-2 Macrophages and the Th1/Th2 Paradigm. J Immunol (2000) 164(12):6166-73. doi: 10.4049/ jimmunol.164.12.6166

47. Liu B, Zhang M, Zhao J, Zheng M, Yang H. Imbalance of M1/M2 Macrophages Is Linked to Severity Level of Knee Osteoarthritis. Exp Ther Med (2018) 16(6):5009-14. doi: 10.3892/etm.2018.6852

48. Daghestani HN, Pieper CF, Kraus VB. Soluble Macrophage Biomarkers Indicate Inflammatory Phenotypes in Patients With Knee Osteoarthritis. Arthritis Rheumatol (2015) 67(4):956-65. doi: 10.1002/art.39006

49. Huo LW, Ye YL, Wang GW, Ye YG. Fractalkine (CX3CL1): A Biomarker Reflecting Symptomatic Severity in Patients With Knee Osteoarthritis. J Investig Med (2015) 63(4):626-31. doi: 10.1097/JIM.0000000000000158

50. Bondeson J, Wainwright SD, Lauder S, Amos N, Hughes CE. The Role of Synovial Macrophages and Macrophage-Produced Cytokines in Driving Aggrecanases, Matrix Metalloproteinases, and Other Destructive and Inflammatory Responses in Osteoarthritis. Arthritis Res Ther (2006) 8(6): R187. doi: $10.5772 / 28284$

51. Culemann S, Grüneboom A, Nicolás-Ávila JÁ, Weidner D, Lämmle KF, Rothe T, et al. Locally Renewing Resident Synovial Macrophages Provide A Protective Barrier for the Joint. Nature (2019) 572:670-5. doi: 10.1038/s41586-019-1471-1

52. Zhang F, Wei K, Slowikowski K, Fonseka CY, Rao DA, Kelly S, et al. Defining Inflammatory Cell States in Rheumatoid Arthritis Joint Synovial Tissues by Integrating Single-Cell Transcriptomics and Mass Cytometry. Nat Immunol (2019) 20(7):928-42. doi: 10.1038/s41590-019-0378-1

53. Alivernini S, MacDonald L, Elmesmari A, Finlay S, Tolusso B, Gigante MR, et al. Distinct Synovial Tissue Macrophage Subsets Regulate Inflammation and Remission in Rheumatoid Arthritis. Nat Med (2020) 26:1295-306. doi: 10.1038/s41591-020-0939-8

54. Fernandes TL, Gomoll AH, Lattermann C, Hernandez AJ, Bueno DF, Amano MT. Macrophage: A Potential Target on Cartilage Regeneration. Front Immunol (2020) 11:111. doi: 10.3389/fimmu.2020.00111

55. Fahy N, de Vries-van Melle ML, Lehmann J, Wei W, Grotenhuis N, Farrell E, et al. Human Osteoarthritic Synovium Impacts Chondrogenic Differentiation of Mesenchymal Stem Cells Via Macrophage Polarisation State. Osteoarthr Cartil (2014) 22(8):1167-75. doi: 10.1016/j.joca.2014.05.021

56. Castrogiovanni P, Di Rosa M, Ravalli S, Castorina A, Guglielmino C, Imbesi R, et al. Moderate Physical Activity as a Prevention Method for Knee Osteoarthritis and the Role of Synoviocytes as Biological Key. Int J Mol Sci (2019) 20(3):511. doi: 10.3390/ijms20030511

57. Raghu H, Lepus CM, Wang Q, Wong HH, Lingampalli N, Oliviero F, et al. CCL2/CCR2, But Not CCL5/CCR5, Mediates Monocyte Recruitment, Inflammation and Cartilage Destruction in Osteoarthritis. Ann Rheum Dis (2017) 76(5):914-22. doi: 10.1136/annrheumdis-2016-210426

58. Appleton CTG, Usmani SE, Pest MA, Pitelka V, Mort JS, Beier F. Reduction in Disease Progression by Inhibition of Transforming Growth Factor $\alpha$-CCL2 Signalling in Experimental Posttraumatic Osteoarthritis. Arthritis Rheumatol (Hoboken NJ) (2015) 67(10):2691-701. doi: 10.1002/art.39255

59. Blom AB, van Lent PLEM, Holthuysen AEM, van der Kraan PM, Roth J, van Rooijen N, et al. Synovial Lining Macrophages Mediate Osteophyte Formation During Experimental Osteoarthritis. Osteoarthr Cartil (2004) 12(8):627-35. doi: 10.1016/j.joca.2004.03.003

60. Wu CL, McNeill J, Goon K, Little D, Kimmerling K, Huebner J, et al. Conditional Macrophage Depletion Increases Inflammation and Does Not Inhibit the Development of Osteoarthritis in Obese Macrophage FAS-Induced Apoptosis-Transgenic Mice. Arthritis Rheumatol (2017) 69(9):1772-83. doi: 10.1002/art.40161

61. Chou CH, Jain V, Gibson J, Attarian DE, Haraden CA, Yohn CB, et al. Synovial Cell Cross-Talk With Cartilage Plays a Major Role in the Pathogenesis of Osteoarthritis. Sci Rep (2020) 10(1):1-14. doi: 10.1038/ s41598-020-67730-y

62. Wojdasiewicz P, Poniatowski ŁA, Szukiewicz D. The Role of Inflammatory and Anti-Inflammatory Cytokines in the Pathogenesis of Osteoarthritis. Mediators Inflamm (2014) 2014:1-19. doi: 10.1155/2014/561459

63. Dell'Isola A, Allan R, Smith SL, Marreiros SSP, Steultjens M. Identification of Clinical Phenotypes in Knee Osteoarthritis: A Systematic Review of the Literature. BMC Musculoskelet Disord (2016) 17(1):1-12. doi: 10.1186/s12891-016-1286-2 
64. Fleischmann RM, Bliddal H, Blanco FJ, Schnitzer TJ, Peterfy C, Chen S, et al. A Phase II Trial of Lutikizumab, an Anti-Interleukin- $\alpha / \beta$ Dual Variable Domain Immunoglobulin, in Knee Osteoarthritis Patients With Synovitis. Arthritis Rheumatol (2019) 71(7):1056-69. doi: 10.1002/art.40840

65. Blair JPM, Bager C, Platt A, Karsdal M, Bay-Jensen AC. Identification of Pathological RA Endotypes Using Blood-Based Biomarkers Reflecting Tissue Metabolism. A Retrospective and Explorative Analysis of Two Phase III RA Studies. PloS One (2019) 14(7):e0219980. doi: 10.1371/journal.pone.0219980

66. Hinks TSC, Brown T, Lau LCK, Rupani H, Barber C, Elliott S, et al. Multidimensional Endotyping in Patients With Severe Asthma Reveals Inflammatory Heterogeneity in Matrix Metalloproteinases and Chitinase 3Like Protein 1. J Allergy Clin Immunol (2016) 138(1):61-75. doi: 10.1016/ j.jaci.2015.11.020
67. Svenningsen S, Nair P. Asthma Endotypes and An Overview of Targeted Therapy for Asthma. Front Med (2017) 4:158. doi: 10.3389/fmed.2017.00158

Conflict of Interest: The authors declare that the research was conducted in the absence of any commercial or financial relationships that could be construed as a potential conflict of interest.

Copyright (c) 2021 Thomson and Hilkens. This is an open-access article distributed under the terms of the Creative Commons Attribution License (CC BY). The use, distribution or reproduction in other forums is permitted, provided the original author(s) and the copyright owner(s) are credited and that the original publication in this journal is cited, in accordance with accepted academic practice. No use, distribution or reproduction is permitted which does not comply with these terms. 